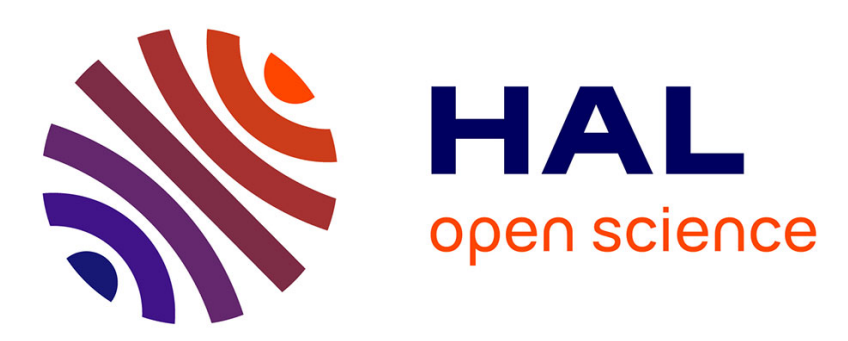

\title{
Prendre ses médicaments/prendre la parole: les usages des médicaments par les patients dans les listes de discussion électroniques
}

\author{
Madeleine Akrich, Cécile Méadel
}

\section{- To cite this version:}

Madeleine Akrich, Cécile Méadel. Prendre ses médicaments/prendre la parole: les usages des médicaments par les patients dans les listes de discussion électroniques. Sciences Sociales et Santé, 2002, 20 (1), pp.89-116. halshs-00119486

\author{
HAL Id: halshs-00119486 \\ https://shs.hal.science/halshs-00119486
}

Submitted on 10 Dec 2006

HAL is a multi-disciplinary open access archive for the deposit and dissemination of scientific research documents, whether they are published or not. The documents may come from teaching and research institutions in France or abroad, or from public or private research centers.
L'archive ouverte pluridisciplinaire HAL, est destinée au dépôt et à la diffusion de documents scientifiques de niveau recherche, publiés ou non, émanant des établissements d'enseignement et de recherche français ou étrangers, des laboratoires publics ou privés. 


\title{
Prendre ses médicaments / prendre la parole : les usages des médicaments par les patients dans les listes de discussion électroniques
}

\author{
Madeleine Akrich, Cécile Méadel
}

\begin{abstract}
Résumé
Quelle place les patients accordent-ils au médicament dans la manière dont ils envisagent leur maladie et leurs rapports aux médecins? Cet article s'appuie sur un matériau original : les contributions de patients à des listes de discussion sur internet. Il met en évidence d'une part, la grande variété des aspects pris en compte par les patients dans leurs échanges sur le médicament, et d'autre part, l'existence de rapports aux médicaments spécifiques de la maladie traitée : dans les trois cas étudiés (cancer, maladie de Parkinson, et fibromyalgie), l'on voit comment, au travers du médicament, se redéfinissent la maladie, le patient, la médecine... en relation avec des éléments hétérogènes comme l'organisation des soins, le partage des compétences entre médecins, l'état des connaissances sur la maladie, sur les traitements, la nature même des traitements.
\end{abstract}

\section{Abstract}

What role does medication play in patients' perception of their disease and their relations with doctors? This article draws on original material: patients' contributions to discussion groups on the Internet. It highlights first the wide variety of considerations taken into account by patients in their discussions on medication and, second, the existence of relations with medication peculiar to the disease in question. In the three cases studied (cancer, Parkinson's disease, and fibromyalgia), we see how, through medication, the disease, patient and medication are redefined in relation to varied elements such as the organization of health care, the sharing of competencies between doctors, the state of knowledge on the disease and its treatment and, lastly, the very nature of treatments.

\section{Mots-Clefs}

Médicament, internet, patients, usages, parkinson, cancer, fibromyalgie

Le rapport des patients aux médicaments a fait l'objet d'une littérature sociologique importante, à la mesure des enjeux économiques et de santé publique qui lui sont associés. De façon générale, on observe une certaine convergence entre les préoccupations des acteurs professionnels du domaine (Meyer, 1984) - médecins, laboratoires, gestionnaires des systèmes de santé - et les questions abordées par les

\footnotetext{
* Centre de Sociologie de l'Innovation, ENSMP, 60 bd Saint Michel, 75006 Paris
} 
chercheurs: l'observance et le respect des posologies, la prévention de l'automédication et de la surmédication sont au cœur de ces différents travaux qui ont souvent pour objectif d'analyser les raisons pour lesquelles, considérées du point de vue des professionnels, les conduites des patients ne sont pas toujours optimales, et de suggérer des voies d'amélioration de ces conduites.

Dans un premier ensemble de recherches, l'analyse se concentre sur l'ensemble des conditions préalables qui assurent le bon déroulement d'un scénario thérapeutique déterminé par le médecin : sont ainsi privilégiés le rôle du nom du médicament et de la forme galénique (Lambert et al., 2001), l'appui que peut apporter la famille ou l'entourage du patient, et enfin l'interaction du patient avec le médecin et l'information délivrée par ce dernier sur le traitement (Homedes et Ugalde, 2001 ; Makoul, et al., 1995). Dans cette optique, le malade est considéré comme passif, le seul problème étant d'arriver à lui faire comprendre la nécessité du traitement et de faciliter sa réalisation.

Un second ensemble de travaux donne un peu plus d'épaisseur au patient: il n'est plus simplement envisagé comme récipiendaire des prescriptions mais, sans être encore un véritable partenaire, peut avoir un point de vue légitime bien que non médical sur le traitement : parce que celui-ci suppose un certain investissement de sa part, il est nécessaire de tenir compte de ses contraintes propres et de son système de représentations (Collin, 1999). Est ici envisagée la question de l'implication du patient dans les décisions thérapeutiques et des " ressources" cognitives (Baril, 1981), sociales, sexuelles (Baumann et al., 1996) et culturelles (Bajos et Bozon, 1999 ; Fainzang, 2001) (Haxaire et al., 1999) qu'il mobilise dans la réalisation du traitement.

Enfin, d'autres auteurs se situent d'emblée du côté du patient et s'intéressent à la manière dont le médicament s'intègre dans leur quotidien et participe de leur mode de vie avec la maladie (Adams et al., 1997), (Mol et Law, à paraître). Dans cette optique, le patient peut se voir accorder un rôle assez actif, en particulier de régulation des dosages médicamenteux dans le cas de pathologies chroniques comme l'asthme ou le diabète. (Willems, 1995 ; Pollock and Grime, 2000)

Ces travaux se situent dans une vision plutôt classique de la médecine et ne bousculent pas l'organisation communément admise du partage des tâches qui s'effectue autour du médicament: la recherche permet de produire de nouvelles substances, mises en forme et testées en association étroite avec l'industrie pharmaceutique; ce travail aboutit à la commercialisation de médicaments auxquels sont associées des indications thérapeutiques définies; celles-ci servent de base aux prescriptions des médecins et des pharmaciens, prescriptions auxquelles les patients sont censés se conformer. Bien entendu, le médicament a besoin d'un soutien constant pour effectuer ce parcours, et au delà des efforts déployés par les médecins pour faire adhérer le patient à son traitement, tout un ensemble de dispositifs accompagnent le médicament - documentation des laboratoires, Vidal, ordonnances, notices, etc. - au long de ses déplacements (Dupuy et Karsenty, 1974 ; Akrich, 1996 ; Maurain, 1996). Mais quels que soient les petits réaménagements effectués par les uns et les autres, la trajectoire linéaire du médicament décrit un espace à peu près stabilisé, dans lequel patients et chercheurs occupent des positions distantes, où les patients ne peuvent guère bousculer les médecins et où les 
pathologies elles-mêmes constituent des repères fixes indépendants des médicaments.

L'émergence de nouveaux acteurs dans cet espace, au premier chef les associations de patients, est venu chambouler ce paysage tranquille: des travaux montrent ainsi que des patients sont susceptibles d'intervenir sur les politiques de recherche (Rabeharisoa et Callon, 1999), de mettre en cause les protocoles des essais cliniques, de contester l'efficacité de certains médicaments, de s'immiscer dans l'établissement du diagnostic, de prendre position sur la définition de la maladie et des formes de traitement (Pignarre, 1999; Ehrenberg, 1999; Dalgalarrondo et Urfalino, 2000). La mise à disposition d'informations médicales sur internet concourt à renforcer leur pouvoir d'intervention en accroissant leur potentiel d'expertise.

Au delà de ces formes très organisées d'intervention des patients, qu'en est-il du rapport $\mathrm{du}$ patient individuel au médicament? Ce mouvement a-t-il des répercussions à l'extérieur du monde associatif ou assiste-t-on simplement à une sorte de "professionnalisation" d'un patient-expert, qui laisserait intouchées les relations installées traditionnellement dans la médecine? Quelle place les patients accordent-ils au médicament dans la manière dont ils envisagent leur maladie et leurs rapports aux médecins?

Pour répondre à ces questions, nous avons choisi de nous intéresser aux listes de discussion constituées à travers la messagerie électronique. Ces listes supposent une démarche volontaire d'adhésion : seuls les abonnés reçoivent les messages de la liste qui est alimentée par leurs contributions volontaires ${ }^{1}$, soit directement, soit avec le filtre d'un animateur. A la différence des forums sur Internet, les listes de discussion sont, du fait qu'elles se construisent un espace propre, propices à la constitution d'un collectif, c'est-à-dire d'un groupe de personnes relativement soudées, avec des rôles progressivement définis (l'expert, le modérateur, le poète, la spécialiste du traitement X...).

Dans la quasi-totalité des cas, les listes se définissent par un intérêt commun pour une pathologie ou pour des questions relatives à la santé (par exemple le cancer, les maladies orphelines, mais aussi la grossesse, l'allaitement ou les problèmes de poids). Elles rassemblent des patients ou des parents de patients ${ }^{2}$, avec éventuellement la participation de professionnels. L'existence de cet intérêt commun pour un problème de santé définit le format des discussions: les fils de discussion $^{3}$ sont souvent longs, détaillés, pluriels ; les invectives sont rares ou vite marginalisées; les appels sont rarement laissés sans réponse. Ce collectif s'inscrit dans la durée, ce qui permet d'étudier les débats dans la continuité.

Dans le cadre d'un travail portant sur la constitution des collectifs autour des questions de santé ${ }^{4}$, nous nous sommes abonnées à la mi-1998 à une trentaine de liste, dont la moitié est animée par des patients. C'est à ces dernières que nous nous

\footnotetext{
${ }^{1}$ Tous n'envoient pas de messages ; ceux qui sont invisibles dans les discussions, appelés “ lurkers " ou "voyeurs" représentent entre 10 et $50 \%$ environ des abonnés, selon les listes.

${ }^{2}$ Pour simplifier, nous parlerons des patients en incluant les locuteurs qui interviennent comme parents de malade.

${ }^{3}$ On appelle fil de discussion l'ensemble des messages dont le sujet est identique.

${ }^{4}$ Ce travail a fait l'objet d'un soutien financier du CNRS et de France-Télécom R\&D.
} 
intéressons ici. Le degré d'activité de ces listes diffère énormément, de quelques centaines à six mille messages par an. Pour cet article consacré au médicament, nous avons extrait (par une procédure automatique de recherche) tous les messages employant les termes "médicament" et "traitement". Ainsi, ont été dépouillés plusieurs centaines de messages.

Ce matériau présente deux caractéristiques originales. Tout d'abord, il ne s'agit ni d'une parole retranscrite, ni d'un écrit formalisé mais d'une correspondance informelle qui a un émetteur individuel et un destinataire collectif, chaque membre de la liste étant susceptible d'être englobé dans les deux catégories. L'écriture y est facile au point qu'un style semi-oral est rapidement adopté par les utilisateurs : ce matériau permet de saisir le vif d'une discussion collectivement élaborée, certes par écrit, mais sans les lourdes médiations de l'écrit ordinaire (Akrich et alii, 2001).

En second lieu, ce matériau n'a pas été produit pour cette enquête, il existe en dehors de notre problématique. La question du " médicament " n'y fait donc pas l'objet d'une co-construction par le chercheur et les acteurs (comme dans le cas d'un entretien), elle n'est pas non plus formatée par un cadre lourdement chargé comme celui de la consultation observée par un sociologue, ni "totalisée" à des fins stratégiques comme dans les compte-rendus de réunions d'associations, mais est saisie dans les relations qu'établissent les locuteurs entre eux. Les interrogations y sont amenées par les participants eux-mêmes. S'agissant de rendre compte des relations que le patient entretient avec le médicament et du rôle que "l'opérateur " médicament joue dans les rapports individuels que chaque patient entretient avec les médecins et la maladie, ce point nous paraît crucial. Bien évidemment, l'appartenance à la liste transforme ces relations, de même que l'entretien avec le sociologue est susceptible de modifier le point de vue de l'interviewé, ne serait-ce que par l'effet de la verbalisation et du travail de rationalisation qu'il suppose de sa part sans parler des mises en relation nouvelles suggérées par l'intervieweur. Un des points essentiels de notre analyse sera que cette transformation s'opère au travers de la confrontation d'expériences individuelles de patients qui, dans leur très grande majorité, ne participent pas à d'autres collectifs liés à leur maladie, tels les mouvements associatifs.

\section{Le médicament comme mode de circulation}

Dans cette première partie, nous allons considérer l'ensemble des listes de patients et décrire les différentes modalités d'apparition des questions relatives aux médicaments. Tout d'abord, nous envisagerons les aspects formels qui renvoient aux types d'engagement des personnes dans le collectif, puis nous nous intéresserons aux thématiques auxquelles réfèrent les messages sur le médicament, qui elles, renvoient aux modalités d'insertion du médicament dans le monde extérieur à la liste.

Les messages prennent essentiellement trois formes rhétoriques: le communiqué, le récit biographique et le débat. La première relève de la communication d'informations, non spécifiques à la personne et à son parcours médical. Le message peut être un simple communiqué faisant connaître par exemple la mise sur le marché d'une nouvelle substance, traduisant un article scientifique ou donnant l'adresse d'un médecin. La liste est ici envisagée comme un 
espace de circulation des connaissances, dans lequel les différents locuteurs occupent a priori des positions équivalentes. La deuxième forme est celle du récit biographique : le locuteur raconte son parcours thérapeutique, ou un épisode de sa trajectoire : par exemple, les effets d'un nouveau traitement. Ce type de récit peut monter en généralité et proposer un message valable en dehors du cas individuel, dans une sorte de croisade commune ou plus modestement dans le but de construire collectivement un référentiel. Il s'agit ici de partir des expériences de personnes saisies dans leur singularité, cette mise en commun des singularités pouvant éventuellement déboucher sur une construction collective de leur identité de patients. Dernière forme rhétorique, le débat ou la controverse fait intervenir plusieurs locuteurs avec des points de vue différents. Les échanges peuvent être si vifs qu'ils conduisent à l'exclusion volontaire ou à la démission silencieuse des hérauts de la position minoritaire ; ou alors contraindre le groupe à formuler une position commune au moins minimale sur le problème en cause. Ces trois formes apparaissent comme des idéaux types entre lesquels un même fil de discussion navigue : une des caractéristiques des listes réside dans la très grande fluidité des échanges qui autorise des changements de registre ailleurs plus problématiques. Le passage et l'articulation possibles entre l'individuel et le collectif, entre le particulier et le général... nous paraissent être une des innovations majeures liées au mode de fonctionnement de ce médium.

Les échanges livrent au lecteur une pluralité d'analyses sur le médicament, techniques, hygiéniques, médicales, économiques... déclinées au regard de la préoccupation de l'usager. Quatre thématiques principales peuvent être relevées. Les deux premiers thèmes sont relativement attendus: les locuteurs considèrent tout d'abord le médicament comme objet politico-économique ; ils s'intéressent aux stratégies des laboratoires pharmaceutiques, à la disponibilité des produits selon les pays; ils réagissent aux annonces publiques, établissent des comparaisons internationales et tentent parfois de faire pression pour obtenir une mise sur le marché plus rapide ou une meilleure prise en charge par les assurances sociales.

Par ailleurs, le médicament est envisagé comme objet scientifique: les contributions de la liste finissent par constituer une impressionnante accumulation - accentuée par le recrutement international des listes - d'informations provenant de la littérature scientifique et généraliste, des communiqués pharmaceutiques, des sites, des praticiens, etc.

Le troisième thème, le médicament comme expérience partagée, donne lieu à de nombreux messages et couvre une multitude d'aspects. Les posologies fournissent des éléments aisément comparables: les locuteurs échangent leurs menus de médicaments, leurs doses, le nombre de prises et les rapprochent de leurs symptômes. La prescription peut y perdre quelque chose de son caractère intouchable; elle est affirmée comme le choix d'un médecin, d'un type de traitement, d'un système de santé publique. Ces comparaisons ne s'arrêtent pas à la prescription du médecin, elles concernent également les différentes façons dont le patient gère son traitement: quels moyens, par exemple, mobiliser pour ne pas oublier ses médicaments lorsqu'il y a une multiplicité de prises ? Ou comment faire prendre des pilules amères à des enfants récalcitrants ? Le tout de la relation au médicament n'est plus dans l'ordonnance ; les débats montrent leur mise en vie, la façon dont les patients s'en saisissent et s'y adaptent. 
Les comparaisons portent encore sur les effets- primaires et secondaires des médicaments. Ceci oblige les locuteurs à se positionner dans la figure stabilisation/guérison/soulagement: pour produire une évaluation qui soit communicable aux autres, le locuteur est poussé à préciser en fonction de quels critères il juge le médicament. Très souvent dans les débats, le diagnostic collectif sur les effets d'un traitement se traduit sous la forme d'un discours de la confiance prudente: espoir en un nouveau traitement et circonspection face aux effets d'annonce, mais aussi face à la généralisation de cas. Cette appréciation des effets échappe à l'alternative efficient/impuissant pour avancer vers une analyse en terme bénéfices/contraintes. Car le médicament a aussi des effets secondaires : il agresse les veines, il provoque des nausées, il irrite et démange la peau, il dessèche le visage... conduisant les locuteurs là aussi à des comparaisons ou à des conseils.

Cette approche des effets secondaires engage bien souvent les locuteurs dans notre quatrième thématique de discussion: le médicament comme mode de relation au médecin. D'une part, se pose la question des informations que les professionnels délivrent aux patients: les médecins doivent-ils prévenir les malades des effets secondaires d'un traitement, et si oui, jusqu'à quel degré de précision? Quelle que soit la position adoptée, certains locuteurs se demandent qui, du médecin ou du patient, met en balance les risques et bénéfices du médicament. $\mathrm{D}^{\prime}$ autre part, ces effets secondaires les conduisent à s'interroger sur la compétence des professionnels: dans quelle mesure ces effets sont-ils considérés par les médecins ? Refusent-ils de les prendre en compte parce qu'ils ne relèvent pas de leur spécialité, parce qu'ils les jugent négligeables, parce qu'ils ne les comprennent pas?

Ces questions deviennent d'autant plus cruciales que le patient n'est plus seul à éprouver ces effets secondaires, il les a vécus au travers d'autres expériences qui lui ont donné des mots pour le dire, voire des solutions pour les soulager ou les adoucir. Comment le patient/locuteur peut-il faire partager à son praticien l'expérience d'autres malades transmise à travers les messages de la liste ? En quoi ce caractère " collectif" du patient peut-il agir sur la prescription du médecin ? Nous ne voulons pas dire pour autant que ces listes soient l'unique moyen de "désindividualiser" le patient; le travail des associations en est un autre, dans certains cas beaucoup plus radical (Pignarre, 1999); simplement nous faisons l'hypothèse que les échanges d'informations, les comparaisons de traitement, les partages d'expérience modifient la position du patient et, partant, sa relation à son médecin. Les locuteurs tiennent grossièrement deux types de positions sur cette question: soit ils poussent leurs colistiers à ne pas accepter passivement la prescription de leurs médecins et à faire entendre leurs doléances; soit ils recherchent une façon de construire un partenariat équilibré entre patient et médecin et réfléchissent aux conditions d'une telle mise à parité. Nous reviendrons plus loin sur les dispositifs conçus par certaines listes dans cet objectif.

En un sens, certaines préoccupations des patients révélées par les listes croisent celles des professionnels: sur les questions débattues actuellement, partage de responsabilité, politique de recherche, économie du médicament, essais thérapeutiques, implication du patient dans le choix des traitements, etc., les patients ont leur mot à dire ou plus exactement essaient d'élaborer un discours, des discours aussi articulés que peuvent l'être ceux des acteurs de la santé, et cela au 
travers d'un médium, la liste de discussion, dont les caractéristiques se prêtent en partie à ce projet : en effet, aucun autre support ne permet à ce point la circulation de textes aux formats aussi variés, de l'article scientifique à l'expérience intime, et leur mise en relation sur un mode à la fois précis et ouvert, dans la mesure où les cadres de l'échange sont peu contraignants. Toute la question est alors de savoir d'une part, comment ces échanges transforment le rapport de chaque individu à la médecine et d'autre part, s'il est possible qu'ils débouchent sur des formes d'action collective, ne serait-ce que par la construction d'un discours cohérent qui inscrive la position des patients dans l'espace public. La réponse à cette dernière question n'a rien d'évident, car le caractère fluide et informel des échanges a son revers, à savoir une robustesse limitée des énoncés, une difficulté à clore les discussions, et une évanescence temporelle.

Pour répondre à ces questions, nous ne pouvons pas nous contenter d'une analyse globale qui agrège les contributions de chaque liste: chacune a son idiosyncrasie et rend visibles des formes très différentes de rapport à la médecine. Ceci est probablement dû à la fois au type de maladie (curable/incurable, évolutive/stationnaire, chronique/aigüe...), à la caractérisation du collectif constitué par la liste de discussion, et à des facteurs externes comme la manière dont la maladie est prise en compte par le système médical. Nous avons choisi de nous intéresser à trois listes - consacrées respectivement au cancer, à la maladie de Parkinson et à la fibromyalgie - qui représentent des positions contrastées du point de vue du rôle accordé au médicament, et de celui du rapport à l'expertise médicale. Concernant des pathologies lourdes, elles ne doivent en aucun cas être considérées comme la source de modèles du médicament qui pourraient être exportés en toute généralité dans d'autres configurations plus banales; en revanche, elles peuvent être le lieu d'innovations radicales qui contribuent à changer en profondeur les prises en charge comme les protocoles de recherche.

\section{Traiter la tumeur/ soigner le malade: médicaments et espaces de compétences dans le cancer}

Cette liste a été créée en 1998 à l'initiative d'une femme alors en cours de traitement; abonnées peu de temps après la création, nous avons pu suivre son développement pendant deux ans et demi. Définie dès le départ comme une liste de soutien mutuel entre malades et proches de malades, elle s'est donnée au fil du temps l'objectif complémentaire de permettre l'échange d'informations pratiques et médicales. Très active - de 200 à 800 messages par mois après une courte période de rodage - elle compte aujourd'hui plus de 70 membres, pour la plupart très assidus. Bien sûr, cette population ne peut en rien être considérée comme représentative de l'ensemble des personnes atteintes de cancer; par ailleurs, pratiquement aucun participant ne mentionne d'affiliation à un mouvement associatif. Sommairement, la liste s'apparente à un groupe de parole qui se renouvelle au fil du temps autour d'un petit ensemble stable de leaders, et dans lequel des personnes réunies par un même problème échangent sur tous les aspects de leur expérience, dans l'espoir que chacun puisse bénéficier du soutien de tous, sans que cet échange ne soit a priori supposé déboucher sur une forme d'action collective. 
Les traitements suscitent des discussions fréquentes qui s'organisent autour de trois axes principaux: les traitements de base du cancer c'est-à-dire la chimiothérapie principalement, les traitements relatifs à d'autres problèmes de santé et leur impact possible sur l'évolution du cancer, les traitements visant à améliorer l'état général et à atténuer les effets négatifs de la chimiothérapie. Nous allons voir qu'à chaque type de traitement est associé un espace dans lequel le partage de l'expertise entre le médecin et le patient est défini de manière spécifique.

\section{La chimiothérapie ou l'espace de l'oncologue}

La chimiothérapie fait l'objet d'un large consensus: l'expertise est ici entièrement du côté des médecins, les patients ne cherchent pas à comparer les protocoles, à s'informer sur ce qui est pratiqué ailleurs, même si l'évolution rapide des protocoles- "qui changent tous les six mois" souligne une participante en ajoutant que "quelque part nous sommes leurs cobayes" - pourrait soulever certaines interrogations. Tout se passe comme si, dans la situation d'urgence vitale dans laquelle se trouvent les patients diagnostiqués, il n'y avait d'autre solution que de s'en remettre aux médecins, en essayant malgré tout de comprendre le traitement, davantage pour se l'approprier, s'y préparer au mieux que pour développer un point de vue critique à son égard. Les débats portent sur les différences éventuelles d'expertise des établissements qui prennent en charge les cancéreux et en aucun cas sur les traitements eux-mêmes, ni même sur les médecins considérés individuellement: seules sont en discussion les formes d'organisation les plus aptes à délivrer une médecine efficace.

Ceci étant, une analyse attentive montre que cette adhésion "spontanée" des patients aux traitements proposés est renforcée par la liste, et même érigée en norme au travers des interventions des leaders. En premier lieu, la non-expertise des patients sur ce sujet est réaffirmée assez systématiquement. Ainsi, une femme interroge la liste sur les traitements possibles d'un liposarcome rétropéritonéal ; la réponse d'un des piliers de la liste est dénuée de toute ambiguité quant au partage des tâches souhaitable entre médecins et patients :

\footnotetext{
“Qu'est-ce que nous aimerions pouvoir dire que nous savons tout du liposarcome rétropéritonéal, avec ou sans métastases! Malheureusement, il n'y a parmi nous, personne qui soit compétent (à moins qu'un médecin caché en coulisse ne se révèle). La seule chose dont nous soyons capables, c'est d'entendre les questions non-médicales que chacun peut se poser et nous manifester en tant qu'êtres humains, aussi misérable cela soit-il. Les questions médicales ne devraient-elles pas être posées à des médecins?"
}

En second lieu, toute déclaration d'un des membres qui tendrait à accréditer l'idée qu'il est possible de se passer éventuellement de chimiothérapie, voire que $\mathrm{d}^{\prime}$ autres traitements non conventionnels peuvent avoir une efficacité est immédiatement reprise, souvent avec véhémence. Une femme qui se présente aux autres membres raconte son parcours et précise qu'elle a refusé la chimiothérapie, décision validée par son oncologue lequel reconnaît que, dans son cas, les bénéfices attendus d'un tel traitement sont très faibles. Face à la réaction d'une participante pour laquelle ce récit jette un doute sur le bien-fondé des chimiothérapies, la réponse est immédiate : 


\begin{abstract}
"Florence, je suis convaincue qu'il faut prendre les propos de Véronique dans son propre contexte et dans celui-là seulement. A ce que je sache les lymphomes et les leucémies se soignent avec de la chimio, et il y a plein de gens qui en guérissent, mais seulement ceux qui se sont fait soigner."
\end{abstract}

De même, les quelques messages qui font état de traitements alternatifs, suscitent des réactions assez vives: si certains se disent intéressés, d'autres sont scandalisés et menacent de se désabonner; le consensus se reforme alors autour de l'idée que ces traitements constituent peut-être des compléments aux traitements conventionnels, la nature de leur efficacité éventuelle étant d'ailleurs incertaine : certains suggèrent qu'elle puisse être davantage liée à l'aura du prescripteur qu'à la chimie du médicament.

Les seuls espaces sur lesquels les participants s'autorisent une certaine liberté sont ceux qui leur sont ouverts par les médecins : l'installation d'un port-à-cathéter qui permet d'éviter les perfusions à répétition lors de la chimiothérapie est l'un des thèmes récurrents sur lesquels expériences et avis sont échangés; il s'agit ici de faire un arbitrage entre deux possibilités en considérant un ensemble d'éléments qui ne ressortissent que marginalement aux aspects médicaux. Parfois - dans le cas de la prise en charge de la douleur - le patient va au-delà de ce qui lui est a priori proposé et prend l'initiative. Se basant sur sa propre expérience - demande $\mathrm{d}^{\prime}$ anesthésies générales pour des examens invasifs et $\mathrm{d}^{\prime}$ un port-à-cathéter - une femme exhorte les autres participants à réclamer pour eux-mêmes, s'ils le souhaitent, des prises en charge différentes de celles qui sont a priori prévues, ce qui nécessite, dit-elle, une bonne dose de combativité et de persévérance. Mais, ici encore, le partage de compétences entre médecins et patients plus ou moins proposé par les premiers est avalisé par les seconds: les patients peuvent être impliqués dans les décisions dès lors que la médecine à elle seule n’a pas $\mathrm{d}^{\prime}$ argument décisif et qu'il est question d'autres dimensions comme le confort $\mathrm{du}$ patient.

\title{
De l'oncologie aux autres spécialités : expertise contre expertise
}

En revanche, lorsque l'on sort du strict cadre de l'oncologie, les patients peuvent déployer une certaine capacité d'expertise médicale et se mettre à discuter avec les médecins de la pertinence des traitements proposés: beaucoup de femmes s'interrogent sur l'effet potentiel d'un traitement hormonal substitutif de la ménopause. La discussion avec le médecin change ici de sens dans la mesure où à une forme d'expertise médicale, les patientes opposent une autre forme d'expertise médicale et non pas seulement des aspects relatifs à leur vie quotidienne de malade.

"J'ai consulté une spécialiste des maladies osseuses. Elle m'a d'abord fait faire une minéralométrie. Ensuite elle m'a fait doser la D-Pyridioline dans les urines et l'ostéocalcine dans le sang.. Ces valeurs mesurent la vitesse à laquelle on "perd" l'os et celle à laquelle on en "refait". Je viens de refaire ces examens et ma perte osseuse s'est bien calmée. Elle m'a dit que je pouvais tranquillement m'abstenir de traitement hormonal pour le moment et refaire une minéralométrie dans 3 ans. J'ai donc résisté au diktat du gynécologue qui me menaçait de l'Alzheimer, des vertèbres qui allaient se déliquescer, du cœur qui allait s'encrasser, etc. Le THS est une issue de santé publique, tout comme les vaccins; c'est à nous de juger de son opportunité en tenant compte de nos facteurs de risques personnels. Je m'en contrebalance du coût annuel des fractures du col du fémur si je sens dans mes entrailles que je risque bien plus de faire un cancer du sein qu'une fracture du col! " 
Impossible ici de postuler l'existence d'une attitude unique à l'égard du médicament et du monde médical qui obéirait à des déterminants socioculturels placés du côté du patient. Les mêmes patients qui laissaient toute latitude à l'oncologue quant à la détermination des traitements adéquats se plongent dans l'analyse détaillée des argumentations médicales quand ils sortent de la cancérologie et offrent une résistance forte à d'autres médecins. Sans doute faut-il voir dans leur attitude " accommodante" à l'égard de l'oncologie en partie le résultat de l'organisation des soins en France qui se caractérise, entre autres choses, par des tentatives répétées pour définir des protocoles rigoureux, applicables en tout point du territoire: le travail de confrontation des différentes stratégies thérapeutiques se ferait en amont, entre professionnels, sans participation des patients ${ }^{5}$. En même temps, la distance critique qui se manifeste dès que l'on quitte la sphère cancérologique est probablement pour certains à mettre en relation avec leur parcours de cancéreux : parce qu'ils ont cherché à s'informer sur leur cancer et son traitement-, ils ont été forcés d'entrer dans la littérature médicale, et cet apprentissage, transposé dans des domaines connexes, leur a permis de développer de nouvelles compétences, ici mises à profit.

\section{Prendre soin de soi, le travail du patient}

Si les traitements des tumeurs cancéreuses font l'objet de peu de contestations, il en va différemment en ce qui concerne les " effets secondaires" de la maladie, effets des traitements (nausées, douleur...) mais aussi effets du cancer lui-même (fatigue, angoisses, etc.). Les interventions se font alors plus critiques: les patients reprochent souvent aux médecins leur incapacité à prendre en charge ces problèmes annexes. S'ils ne remettent pas en cause la définition " organique" du cancer - on n'observe aucune tentative de suggérer une explication psychosomatique, si ce n'est sur un mode humoristique - ils englobent en quelque sorte cette définition dans une définition de la "maladie vécue", qui, elle, appartient au registre psychosomatique : lorsque l'angoisse génère des insomnies, celles-ci peuvent avoir des retentissements sur la capacité organique du patient à surmonter la maladie. Laissés sans appui par beaucoup d'oncologues sur ces questions, ils sont en quelque sorte poussés à déployer une certaine activité, soit en consultant d'autres médecins (psychiatre, généraliste, homéopathe), soit en cherchant eux-mêmes des informations sur des traitements d'appoint.

Les discussions montrent un certain consensus sur les médicaments conventionnels : anti-douleurs, anxiolytiques, antidépresseurs, ou... verre de vin rouge sont considérés comme des recours possibles au cas par cas en fonction de l'évaluation que chacun fera du rapport entre ses difficultés, sa souffrance et les risques associés à la prise de médicament. En revanche, les médicaments associés aux médecines douces suscitent des débats beaucoup plus houleux. Grossièrement, deux positions s'opposent: d'un côté, un certain nombre de patients s'insurgent contre le peu de cas qui est fait par les oncologues des médecines non allopathiques et échangent informations sur les médicaments, les posologies, les effets qu'ils observent sur eux-mêmes, les "filières" d'approvisionnement... Ce travail sur les

\footnotetext{
${ }^{5}$ Ce qui est congruent avec (Ménoret, 1999).
} 
médicaments dits d'appoint est le moyen, pour ces malades, de reprendre une sorte de contrôle sur eux-mêmes, leur corps, leur maladie, contrôle qui leur échappe dans le cadre des traitements conventionnels. Au delà du soulagement que ces médicaments leur apportent, il s'agit de s'engager fortement dans sa propre guérison, et à ce titre, les difficultés rencontrées dans le recueil d'informations et dans l'approvisionnement font partie intégrante de ce processus :

"Ceux qui font la démarche de "trouver" et de suivre un traitement d'accompagnement ont une attitude volontaire, ont assimilé le fait d'être malade, accepté la chimio, souhaitent en limiter les effets secondaires. Bref, ils ont décidé de se prendre en main, d'être actifs et de lutter. Et la part de volonté face à cette maladie n'est sûrement pas anodine."

De l'autre côté, certains colistiers contestent a priori l'efficacité “ propre" de ces médicaments. Dans leur optique, ceux qui se sortent bien de l'épreuve de la chimiothérapie et plus généralement de la maladie sont susceptibles d'appartenir à deux catégories, ceux qui ont le moral et la chance d'avoir un organisme qui réagit bien aux traitements conventionnels, et ceux pour lesquels l'effet placebo fonctionne, le médicament servant alors de support à une croyance qui s'autoréalise.

De façon à première vue curieuse, les tenants de la médecine allopathique "pure" investissent beaucoup moins de temps et d'énergie dans l'analyse des modalités d'action des substances médicamenteuses que ceux qui font appel aux médecines douces. Tout se passe comme si le fait qu'un médicament soit inséré dans les réseaux de la médecine officielle suffisait à prouver son efficacité ou au moins son innocuité. Ainsi, pour une patiente qui en appelait au sens critique des malades vis à vis des " autres" médicaments, le DHEA change de statut du simple fait qu'il est prescrit par un organisme reconnu.

En contraste avec cette attitude qui consiste finalement à s'en remettre à l'expertise officielle, ceux qui défendent l'utilisation des médecines douces s'appuient sur des arguments de nature scientifique : ils cherchent à démontrer que les effets des médicaments se trouvent bien dans les substances. En dehors de l'homéopathie qui fournit son système d'interprétation propre doté d'une légitimité reconnue même si elle est controversée, les autres médicaments sont passés à un crible de nature allopathique. Ainsi, si le petit lait a des vertus curatives, c'est parce qu'il permet de " lutter contre les radicaux libres ", comme l'atteste " un article du Dr Carol Vachon ". En forçant délibérément le trait, on pourrait dire que les tenants des médecines douces sont encore plus "allopathiques" que les "allopathiques" : le format de la liste leur permet l'accumulation des contributions diverses qui apportent à des choix personnels l'épaisseur et la robustesse d'une argumentation générale.

Les malades du cancer nous rendent visibles la segmentation de l'espace médical opérée au travers du médicament: selon que l'on se trouve dans le domaine de l'oncologie, ou dans celui de la gynécologie, ou encore dans l'espace du patient (tel qu'il résulte des choix qui ont été faits par les professionnels de santé sur ce qu'ils considèrent relever de leurs soins), le rapport au médicament et surtout la distribution de l'expertise diffèrent radicalement. 


\section{De la co-gestion de la maladie à la recherche : le médicament comme opérateur dans le réseau des parkinsoniens}

La liste consacrée à la maladie de Parkinson est une liste extrêmement active qui compte 300 inscrits en juillet 2001, alors qu'elle existe depuis janvier 1997. Abonnées en août 1998, nous avons reçu depuis près de vingt mille messages, soit une moyenne de plus de 120 messages par semaine. La participation est élevée : $40 \%$ des locuteurs interviennent tous les mois ; la liste est aiguillonnée par un groupe d'une vingtaine de leaders très présents; elle évolue puisqu'elle intègre sans arrêt de nouveaux membres, dont certains deviennent des locuteurs actifs : près des deux tiers de ceux qui ont une participation durable (plus de six mois de présence) interviennent sept mois sur dix.

Les médicaments suscitent de nombreux messages: nous verrons que l'adaptation des médicaments est un problème permanent en raison des caractéristiques de la maladie et que la liste vise à équiper les patients afin qu'ils puissent participer activement au suivi de leurs traitements.

Le sort des parkinsoniens - tel qu'il apparaît dans la liste elle-même - est inéluctablement lié aux médicaments, et ce à un double titre. D'une part, c'est "l'invention" de la L-Dopa à la fin des années 60 qui a transformé la vie des patients: la maladie demeure incurable, mais elle n'est plus inéluctablement mortelle en une dizaine d'années ; son évolution est ralentie et les symptômes sont moins handicapants. D'autre part, la principale manière de savoir si une personne est atteinte de Parkinson est d'observer les effets d'un traitement à base de dopamine: en effet, cette maladie est difficile à diagnostiquer tant les symptômes sont variables et pour certains communs à d'autres pathologies. Sans dopamine, les cellules du cerveau ne peuvent plus communiquer, ce qui entraîne des troubles du système nerveux, lesquels se manifestent par des rigidités musculaires, des tremblements, des lenteurs de mouvements. Devant ce tableau, la perspective des patients est claire: ils ne peuvent vivre sans apport de dopamine et cette prescription émane nécessairement d'un médecin.

Cependant, les médicaments qui apportent la dopamine au système nerveux entraînent en même temps une série d'effets secondaires qui peuvent aller du déplaisant à l'insupportable. Aussi l'objectif des traitements est-il de maintenir au plus bas la dose de dopamine absorbée quotidiennement. Mais la maladie et le traitement sont tous deux évolutifs : l'état dégénératif a tendance à s'accentuer et le traitement perd de son effet. Après une première phase de traitement, appelée cruellement " la lune de miel" pendant laquelle l'effet des traitements est quasi " miraculeux", les réactions deviennent imprévisibles, les effets secondaires insupportables. Il faut alors passer à un second stade du traitement qui adjoint une autre classe de médicaments que les patients appellent des "économiseurs" de dopamine. La maladie est en outre hiératique: les patients racontent ainsi des blocages brusques qui les ont empêchés de redémarrer leur voiture à un feu de circulation, les blocages inattendus qui leur donnent la " démarche hésitante d'un ivrogne", les tremblements brutaux qui rendent leur écriture illisible, les confusions intellectuelles... Les médicaments jouent un rôle central car c'est seulement par un juste dosage que les patients peuvent espérer réduire les manifestations les plus handicapantes et les membres de la liste s'accordent sur le fait que cet équilibre ne peut être atteint sans leur participation active : 


\begin{abstract}
“ La variabilité des symptômes étant le quatrième signe de la maladie, rien n'est jamais acquis et ce qui est vrai aujourd'hui sera peut-être faux demain. C'est une démarche volontariste du malade qui apprend à se connaître, à connaître sa maladie et peut ainsi mieux se prendre en charge."
\end{abstract}

Le problème qui se pose aux malades est de savoir comment aboutir au traitement idéal dans une situation d'incertitude; rien en effet n'est fixe: la maladie, l'effet des traitements, les facteurs qui agissent sur le traitement (psychologiques, alimentaires...), les médicaments, tout évolue dans l'univers du parkinsonien. Ce réglage des posologies fait l'objet de très nombreux messages qui s'interrogent sur les dosages, le cocktail de médicaments et les temps de prise. Une locutrice parle du "tâtonnement épuisant et décourageant ". L'un des objectifs que s'est construit la liste, au travers des interventions récurrentes de leaders, consiste justement à accompagner les patients et leurs familles dans cette expérimentation permanente non seulement en s'obligeant à fournir des réponses à chaque question mais aussi en proposant une conception arrêtée du rôle des médicaments et un modèle de relation avec le corps médical.

Le groupe construit autour de la liste impose collectivement une " morale du médicament" (titre d'un message), morale consensuelle qui explicite ses propres règles : $\mathrm{d}^{\prime}$ abord le patient est " responsable" de son traitement dans la mesure où il doit être très attentif aux manifestations et aux variations de sa maladie, sachant que chaque cas est spécifique :

" Oui, on doit demander que le neurologue consacre un peu plus de temps pour une consultation. Oui, on doit recevoir des réponses à nos questions et non pas des dérobades. Oui, on doit changer de médecin si on n'a pas confiance. Mais si nous avons des exigences légitimes, nous avons aussi des devoirs : être coopératif et ne pas faire la grimace lorsqu'il faut remplir un tableau toutes les demi-heures et s'observer à la loupe. C'est pour nous que nous le faisons, pour permettre à notre spécialiste de mieux cibler notre traitement."

Ensuite, la prescription doit être le résultat d'un travail commun entre le patient et son médecin. Alors que les conseils thérapeutiques, les comparaisons de prescriptions, les signalements de médicaments abondent dans la liste, ils réprouvent fortement l'automédication considérée comme dangereuse. Enfin, l'objectif commun est de prendre le minimum de médicaments mais de prendre tous les médicaments nécessaires. Ainsi un patient qui se plaint d'insomnie mais refuse de prendre des somnifères se fait rappeler à l'ordre par plusieurs correspondants et finit par avouer, penaud, qu'il a tort.

Cette position forte trouve une réalisation pratique dans un outil proposé par le modérateur-créateur de la liste, un ingénieur, lui-même malade, avec l'aide de “l'expert", un médecin dont l'épouse est atteinte. Ce logiciel d'aide à la médication permet de suivre la progression de la maladie et d'adapter le traitement aux variations de l'état du malade. Le patient doit fournir peu d'informations pour que le logiciel puisse fonctionner ("l'âge" de sa maladie, sa posologie, et les symptômes); il obtient alors une courbe de médication qui doit lui permettre d'améliorer son traitement. Ce logiciel a subi une série d'épreuves et d'améliorations successives, il a été testé auprès d'un nombre élevé de malades, rencontrés par la liste, sur le web, ou dans des réunions externes. Il s'adapte à toutes les situations locales puisque les différentes formes galéniques (internationales) ont été intégrées. Les laboratoires pharmaceutiques ont été associés à ses mises à jour en fournissant au concepteur des notices sur la composition des médicaments au fur 
et à mesure des nouvelles arrivées sur le marché. Les patients considèrent qu'il a même permis de valider certaines connaissances sur la maladie : de leur point de vue, il a montré par exemple que la quantité de médicaments nécessaires est liée non à l'âge du patient mais à l'ancienneté de la maladie. Ce logiciel est donc envisagé, au moins par les locuteurs de la liste, comme un outil fiable d'aide à la médication qui débouche sur une meilleure qualité de vie pour les patients, en optimisant la prise de médicaments. Nous retrouvons ici l'idée, développée sur le cas des maladies chroniques (Pollock et Grime, 2000 ; Willems, 1995), que le patient peut jouer un rôle majeur dans la régulation de son traitement: l'innovation radicale $q u^{\prime}$ introduisent les parkinsoniens tient au fait que cette autorégulation passe par la médiation d'un outil élaboré par les patients eux-mêmes, ce qui les met en quelque sorte à parité avec le monde médical.

Cependant, cet outil mis à leur disposition n'est pas abandonné à leur libre utilisation. D'abord, les animateurs conseillent fortement aux utilisateurs novices de passer dans un premier temps par un des experts pour faire établir leur première courbe médicamenteuse accompagnée d'une fiche de conseils. Ensuite, même les utilisateurs plus chevronnés sont priés de faire connaître leurs résultats et leurs interprétations, non seulement afin d'accroître la compétence collective mais aussi pour vérifier que les conséquences tirées sont judicieuses et pour mieux comprendre tout ce que l'on peut attendre d'un dispositif qui se veut toujours expérimental. Par sa " rigidité" - le patient ne peut pas modifier les paramètres du logiciel - et les contraintes imposées sur son utilisation, le logiciel incorpore en quelque sorte la liste comme collectif; par la généralisation qu'il permet, il constitue un point d'arrêt dans le flux ininterrompu des messages de la liste.

Quelle place le logiciel attribue-t-il au médecin? Les locuteurs refusent collectivement de s'en remettre aveuglément à leur neurologue : l'expérience de chaque patient est considéré comme intransmissible à qui n'a pas vécu une expérience similaire : la maladie est trop "capricieuse", "inattendue", " incompréhensible".Leur " expertise" personnelle est difficilement transmissible également à cause de la maladie elle-même et de ses problèmes de blocage intellectuel ou de défaillance de la mémoire. L'asymétrie entre la position du patient, désarmé et impuissant, leur est d'autant plus insupportable qu'elle est pour eux un des symptômes de la maladie :

\footnotetext{
" Partant du constat 1) que le neurologue est toujours débordé ou très pressé, 2) que sa compétence n'est pas mise en doute a priori, qu'en particulier il est admissible que "son coup d'œil" professionnel soit souvent suffisant pour avoir une idée de l'état d'un malade anciennement connu et vu régulièrement 3 ) que son examen clinique est en conséquence souvent succinct, l'interrogatoire encore plus et que la conclusion habituelle est "Pas de changement, continuez le traitement, à dans quatre mois" et donc que 4) le Parkinsonien est souvent frustré de n'avoir pu poser toutes les questions souhaitées et qu'il sort de sa visite chez le neurologue un peu déçu, il me semble souhaitable d'envisager une autre forme de rapports médecin-malade, d'autant plus que par sa maladie même le parkinsonien est lent dans sa réflexion et ses réactions."
}

Le patient ne gagne donc pas son indépendance en se passant de son médecin, mais plutôt en produisant les bases d'un travail commun sur son traitement, au travers de l'objectivation permise par le logiciel.

Les compétences que les locuteurs ont collectivement acquises en scrutant la littérature, en s'associant à des médecins et aux laboratoires peuvent ainsi être 
mobilisées par le patient dans ce moment délicat et "frustrant" de la consultation. Comment collaborer avec le médecin et établir des relations de co-prescription qui associent le médecin et le malade ? En faisant reconnaître les expérimentations qu'il a accompli sur son propre corps :

"Il est important que le patient tire les leçons de son expérience, apprenne quels symptômes indiquent qu'il a pris trop de médicaments ou qu'il a besoin de plus de médicaments. Les premières années de l'utilisation de la L.dopa ceci ne semble pas un problème, parce que la fenêtre thérapeutique est encore assez large (la fenêtre thérapeutique est la différence entre la dose minimale qui a un effet thérapeutique et la dose qui a des effets secondaires graves). Cependant, même à ce moment-là, il faut qu'un patient apprenne à connaître cette dose minimale et ne prenne pas plus de L.dopa que nécessaire, d'où le rôle primordial de l'expérimentation. (...) Le neurologue, qui voit le patient une demi-heure tous les trois mois, ne peut pas faire ces expériences lui-même ; le rapport entre le neurologue et le patient doit être considéré comme en partie semblable à celui qui existe entre deux chercheurs, un chercheur senior (le neurologue) et un junior (le patient qui fait l'expérience, mais a besoin de conseils). Si leurs rapports sont définis de cette manière, le neurologue ne doit pas se sentir menacé dans ses compétences."

On le voit, la redéfinition du champ médical proposée, grâce au médicament, par les parkinsoniens de la liste est radicale, en ce sens qu'elle place le patient au centre du réseau, à la fois à un niveau individuel puisque toute prescription ne peut être qu'une co-prescription dans laquelle chacun des partenaires apporte des connaissances spécifiques irremplaçables, et à un niveau collectif puisque le patient devient un opérateur de recherche incontournable. Toute la question aujourd'hui est de savoir dans quelle mesure ils pourront imposer cette redéfinition au delà des frontières de leur groupe étendu à leurs partenaires extérieurs : les médecins, en tant que collectif, sont-ils prêts à admettre une telle révolution?

\section{Le médicament comme mode de constitution de la maladie chez les fibromyalgiques}

Notre troisième liste de discussion est consacrée à la fibromyalgie et au syndrome de fatigue chronique. Créée en mars 2000, elle compte aujourd'hui 140 abonnés et déploie une activité considérable puisqu'en 16 mois d'existence, plus de 7000 messages ont été échangés. Pour des raisons qui sont au cœur de l'analyse que nous allons proposer, la thématique " médicaments" est une des thématiques majeures des discussions à l'intérieur de la liste. Tels qu'ils apparaissent dans les descriptions qu'en font les participants à la liste et dans les informations externes échangées, le syndrome de fatigue chronique (SFC) et la fibromyalgie se caractérisent par une série de symptômes invalidants pour les malades, la liste de ces symptômes étant elle-même encore en débat. Un message de la liste mentionne ainsi pas moins de trente-deux symptômes différents, parmi lesquels intolérance aux efforts, troubles de mémoire, problème de concentration, douleurs musculaires, sommeil non réparateur, maux de tête etc.. Pour les patients, il semble que la différence entre fibromyalgie et SFC se fasse sur l'absence (SFC) ou la présence (fibromyalgie) de douleurs musculaires intenses. Bref, si la réalité de la maladie est absolument incontournable pour les patients et ceux qui ont à les traiter, sa définition est loin d'être clairement établie, d'autant qu'il ne semble pas y avoir encore de méthode de diagnostic fiable qui reposerait sur l'identification et la reconnaissance de paramètres objectivables, même si certains tests commencent à être pratiqués. La difficulté est redoublée par le fait que l'incertitude la plus radicale 
règne sur la nature des mécanismes en cause dans la maladie. Parmi les "causes" possibles évoquées par la liste, on peut citer : une condition inflammatoire, une condition psychiatrique, une anomalie du sommeil profond, une anomalie du système immunitaire liée ou non à une infection cachée, une intoxication aux métaux lourds, une anomalie dans le métabolisme musculaire, une maladie génétique ... De plus, on ne sait si la maladie résulte d'une conjonction de différents facteurs, ou si certains facteurs ne sont en fait que la conséquence d'autres. Malgré toutes ces incertitudes et en considération du nombre de patients souffrant des “ mêmes" symptômes, l'OMS a reconnu cette maladie en 1992, et les autorités sanitaires françaises ont fait de même en 1996.

\section{De la co-construction des médicaments et de la maladie}

Dans ce contexte, on comprend que l'expérience des patients, et les données produites par leur auto-observation soient cruciales: c'est essentiellement au travers de la description de leurs sensations, de leurs douleurs et de leurs difficultés qu'un diagnostic peut aujourd'hui être porté ${ }^{6}$. Le médicament joue alors un rôle central : c'est la seule prise que le patient a sur sa maladie, à la fois pour essayer de la guérir et pour la connaître.

Première caractéristique du rapport qu'entretiennent les patients au médicament, les médicaments produisent toujours des effets, positifs ou négatifs et ceux-ci sont clairement identifiables. Il n'y a jamais de doute sur ce point, les modifications observées dans leur état - à défaut de pouvoir être imputées à des causes biologiques connues - sont rapportées aux médicaments. L'hypersensibilité à leur corps, que la maladie et leurs efforts longtemps infructueux pour trouver avec les médecins un nom à mettre sur ce qu'ils ressentent ont contribué à développer, est ensuite perpétuellement réactivée dans leur utilisation des médicaments. En voici un exemple :

\footnotetext{
“ J'aimerais savoir si, sur notre groupe, vous avez fait l'expérience de certains médicaments que j'ai pris : 1. substance midodrine commercialisé sous le nom de GUTRON 2,5 mg pour l'hypotension; je devais monter à $6 c p$ par jour mais vertiges, céphalées, nausées, fièvre. Pas de hausse de tension. Ceci à $3 \mathrm{cp} / \mathrm{j}$. J'ai abandonné au bout de 3 semaines. Je ne supportais pas. 2. Substance piracetam commercialisé sous le nom d'AXONYL(...). Mercredi 16, un neurologue de la Pitié m'a prescrit NOOTROPYL $400 \mathrm{mg} 3 \mathrm{cp} / \mathrm{J}$. J'en ai pris 2 le 16, le 17 1cp. Effet foudroyant. Nausées++++, vertiges+++, fièvre et surtout la face $G$ et l'œil G se paralysant..."
}

En second lieu, l'incertitude qui prévaut sur la maladie et ses mécanismes pose un problème particulier de prescription: il ne semble pas exister de médicament spécifique de la fibromyalgie ou du SFC; la seule stratégie consiste à essayer de soigner les symptômes un par un, sans être sûr de l'efficacité de cette méthode dans la mesure où des symptômes semblables peuvent renvoyer à des causes différentes et donc ne pas être sensibles aux mêmes substances. Étant donné la diversité des symptômes ressentis, les patients ingurgitent nombre de médicaments : contre l'hypotension, relaxants, antidépresseurs, anti-inflammatoires, anti-douleurs, stimulants y compris amphétamines, antibiotiques... Ce travail de "ciblage"

\footnotetext{
${ }^{6}$ Même si certaines études relèvent la présence récurrente chez les malades d'une certaine enzyme ou d'hypoglycémie.
} 
médicamenteux est fait à la fois par le médecin et par les patients qui glanent quantité d'informations et échangent expériences et avis.

Au travers des médicaments, auxquels sont associés des indications officielles, se trouve opérée une liaison entre certaines maladies aux mécanismes plus ou moins connus et les symptômes ressentis par les fibromyalgiques. Cette liaison est activée à plusieurs reprises par les patients qui réalisent donc ici un travail inverse de celui qui a permis d'aboutir à la prescription: le symptôme a servi dans un premier temps à trouver le médicament et celui-ci, par sa réaction sur le corps du malade, va valider ou invalider certaines théories possibles de la maladie. Les patients se considèrent comme le siège d'une expérimentation permanente via le médicament, expérimentation personnelle - il s'agit de trouver le mélange spécifique de substances qui permettra de stabiliser leur état - mais aussi expérimentation plus générale dans la mesure où ils essaient d'articuler leur expérience à des théories médicales portées par les médicaments; ici, la sensibilité des patients aux antibiotiques est envisagée comme une validation possible d'une théorie infectieuse du SFC:

\footnotetext{
"Suite aux récents échanges de discussion sur les traitements prescrits contre les infections, je voudrais savoir combien y en a-t-il parmi vous qui vous sentez mieux depuis la prise des antibiotiques? A-t-on noté une certaine amélioration au niveau immunitaire ? Si je pose ces questions, c'est que je suis d'avis que le SFC implique un double problème à savoir 1 . un dysfonctionnement enzymatique et neuro-hormonal, et 2. la présence d'infections chroniques. J'ai acquis la conviction qu'il faut agir à ces 2 niveaux sinon on risque de tomber dans un cercle vicieux, les infections vont réapparaître et se généraliser encore et encore... En terminant, je vous laisse un article qui me semblait très à propos(...)"
}

Ce type d'expérimentation s'apparente à première vue à ce que l'on appelle "l'épreuve thérapeutique": face à des symptômes qui évoquent plusieurs pathologies sans que l'on puisse trancher, le médecin peut fixer le diagnostic en testant successivement l'efficacité de médicaments spécifiques à chaque pathologie. Mais insistons sur la différence radicale qui existe entre les épreuves thérapeutiques classiques et ces expérimentations des fibromyalgiques: dans le premier cas, il s'agit de déterminer si une personne est ou non atteinte par une pathologie définie, alors que dans le second, la pathologie est identifiée, nommée, et les personnes constituent en quelque sorte le milieu d'expression de la maladie que l'épreuve par le médicament va peut-être permettre de mieux comprendre. L'on assiste à un renversement de perspective: il ne s'agit pas de produire de la connaissance sur l'état d'une personne, mais de produire de la connaissance sur une maladie. La liste de discussion, par le type d'échanges et d'accumulation collective d'expériences qu'elle permet, apparaît comme le médium privilégié par lequel peuvent être produites ces connaissances.

Parfois, en revanche, essentiellement pour les antidépresseurs, certains patients refusent activement la liaison qui pourrait être opérée par le médicament, refus qui doit être mis en relation avec une opposition franche à toute idée de psychiatrisation de leur maladie. Leurs souffrances sont réelles, elles prennent racine dans leur corps et non dans leur psychisme. Dans ce contexte, l'utilisation des antidépresseurs, très fréquente, est écartée par certains, ce qui provoque l'indignation d'autres qui développent tout un ensemble d'arguments afin de montrer que l'utilisation d'antidépresseurs n'implique pas la dépression. L'argumentation s'organise autour de deux points principaux. D'une part, les antidépresseurs 
sont prescrits en raison de leurs effets " annexes" et non de leurs effets sur la dépression : "ce médicament a un AUTRE effet, différent de l'effet antidépresseur: il agit sur mon seuil de douleur et améliore mes troubles du sommeil"; et ces effets peuvent être scientifiquement expliqués : "les antidépresseurs sont utilisés pour agir sur la perception de la douleur par le cerveau (à cause de leur action sur la sérotonine et plus globalement les échanges chimiques au niveau du cerveau) ". D'autre part, les posologies utilisées sont plus faibles que dans les traitements de la dépression, prouvant que l'on a affaire à deux médications différentes même si elles s'appuient sur le même médicament. Ce n'est pas pour autant que tout diagnostic de dépression doit être écarté, mais cette dépression est la conséquence de la maladie - " dépression réactive" - et non sa cause.

Nous le voyons au travers de cet exemple des antidépresseurs, les patients développent des théories élaborées des médicaments qui ne peuvent être réduits à la définition officielle de leurs " effets thérapeutiques": ils agissent, c'est indubitable, mais cette action est complexe et le corps des fibromyalgiques rend en quelque sorte visible cette complexité.

Pour les fibromyalgiques et les patients atteints du SFC, le médicament et la maladie s'entredéfinissent : $c^{\prime}$ est par le médicament que s'expérimentent diverses constructions de la maladie, et en même temps c'est par l'épreuve des corps malades que se redéfinissent les propriétés du médicament.

\section{Sortir du médicament?}

Cette attention extrême portée aux effets entraînés par l'ingestion de différentes substances s'étend au delà de l'univers médicamenteux : à défaut d'une théorie opératoire de leur maladie, certains malades se représentent leur propre état comme le résultat d'une hypersensibilité à leur environnement. Du coup, l'alimentation, les modes de vie (sommeil, activités, relations aux autres) deviennent l'objet d'une même circonspection.

Si cet hygiénisme est assez partagé, il est pensé comme complémentaire de la prise médicamenteuse. De façon exceptionnelle, il peut, par une sorte de renversement, conduire à exclure le médicament qui apparaît comme une béquille inefficace et même dangereuse dans la mesure où elle induit des déséquilibres nouveaux et incontrôlés dans une situation déjà périlleuse : l'épuisement des traitements médicaux classiques conduit alors à une nouvelle définition de la maladie, comme conséquence, sur des organismes plus sensibles que les autres, de modes de vie " déréglés" par la civilisation moderne. Ce point de vue est exprimé de manière radicale par un abonné :

\footnotetext{
"Quant à la différence entre une dépression et un SFC, ce sont deux façons du corps d'exprimer son ras-le-bol du régime de vie que nous lui imposons. Ces réactions sont causées par l'intoxication du corps qui n'a plus le temps de se nettoyer normalement parce qu'on ne se repose pas assez et que l'on mange mal, probablement parce qu' on veut prouver trop de choses aux autres ou à soi-même... Le type de maladie qui prend place dépend de nos faiblesses héréditaires. Mais avec une bonne conduite normalement nous n'aurions pas dû tomber malade. Je me méfie des docteurs et des poisons qui guérissent; le corps a une intelligence dans l'auto-guérison qu'il faut apprendre à respecter. J'ai l'impression depuis que je suis malade d'être différent des autres. Cette maladie prend l'allure d'une initiation en profondeur à l'existence, à la vie. Elle me traverse de bord en bord, me transforme et me
} 
porte vers le rivage d'une autre vie, vers de nouvelles perspectives, de nouvelles visions et de nouvelles aventures. De plus en plus, elle prend son sens. Elle devient mon guide, mon alliée vers une nouvelle vie."

L'expérience de la maladie débouche sur une métaphysique, renouant avec certaines philosophies millénaires dans lesquelles le "souci de soi" constituait le moyen d'inscrire la philosophie dans l'expérience quotidienne (Foucault, 1994). L'allégeance de ce locuteur à une forme extrême d'hygiénisme le conduit à prêter une attention de tous les instants à son corps dans ses rapports avec l'environnement et surtout à rejeter toute prise médicamenteuse qui ne ferait que poursuivre le "débalancement" produit par des modes de vie inadéquats. Cette position suscite des réactions très vives - " je sais que mes médocs ne sont pas une camisole de force" affirme une abonnée - de la part des autres membres de la liste dont nous avons vu à quel point leur vie s'organisait autour et par le médicament.

A l'issue de discussions houleuses, "l'hygiéniste" se désabonne : il ne sera resté sur la liste que quelques semaines, mettant en quelque sorte à l'épreuve le rapport des fibromyalgiques au médicament. Mais, en un sens, même l'expérience de ce patient est profondément structurée par le médicament, et pas uniquement par l'échec de ses contacts prolongés et infructueux avec lui : le nouveau rapport qu'il établit avec son environnement a été construit par cet apprentissage particulier aux fibromyalgiques qui ne peuvent envisager autrement la maladie que dans la confrontation de leurs corps avec des substances agissantes.

A l'issue de notre parcours, deux conclusions principales nous paraissent devoir être tirées. Tout d'abord, l'analyse montre qu'il n'y a pas UNE forme de relation au médicament qui serait spécifique à chaque patient et obéirait à des déterminations placées du côté du patient: cette relation ne peut être saisie que dans le rapport construit à la maladie, de même que l'expérience de la maladie passe en grande partie par le rapport aux médicaments. Plus encore, dans le contexte même d'une maladie donnée, les patients sont susceptibles d'entretenir des relations variées aux médicaments, sous l'angle du partage d'expertise entre médecin et patient comme sous celui des modalités par lesquelles se construit l'expérience du médicament, de l'énonciation subjective aux constatations objectivées, de l'expérience incorporée aux connaissances certifiées...

En second lieu, le médicament apparaît comme un opérateur majeur dans la reconfiguration possible de la médecine comme science, des pratiques médicales, des relations médecin-patient ; plus précisément, cette possibilité de transformation tient au fait qu'il permet la liaison entre ces différents aspects, plaçant du même coup le patient au cœur du réseau qu'il contribue à installer, liaison ré-effectuée par l'intermédiaire de ce médium spécifique, la liste de discussion, dont le format autorise la mise ensemble de productions discursives autrement hétérogènes.

\section{Bibliographie}

Adams, S., Pill, R. et Jones, A., 1997, Medication, chronic illness and identity: The perspective of people with asthma, Social Science $\mathcal{E}$ Medicine, 45, 2, pp.189-201. 
Akrich M., 1996, Le médicament comme objet technique, Revue internationale de Psychopathologie, 21, pp.135-158.

Akrich M., Méadel C. et Paravel V., 2001, Le temps du mail écrit instantané ou oral médiat, Sociologie et Sociétés, XXXII/2, pp.153-170.

Bajos, N. et Bozon M., 1999, La sexualité à l'épreuve de la médicalisation : le Viagra, Actes de la recherche en sciences sociales, 128, pp.34-37.

Baril G., 1981, La dimension cognitive du rapport aux médecines parallèles, Culture, 17, 1-2, pp.101-109.

Baumann M., Pommier J. et Deschamps J. P., 1996, Prescription médicale et consommation de psychotropes: Quelques interrogations sur les différences entre hommes et femmes, Cahiers de sociologie et de démographie médicales, 36, 1, pp.63-78.

Collin J., 1999, La construction d'une rationalité : les médecins face à la prescription de psychotropes aux personnes âgées, Sciences sociales et santé, 17, 2, pp.31-50.

Dalgalarrondo S. et Urfalino P., 2000, Choix tragique, controverse et décision publique : Le cas du tirage au sort des malades du sida, Revue française de sociologie, 41, 1, pp.119-157.

Dupuy J.-P. et Karsenty S., 1974, L'invasion pharmaceutique, Paris, Seuil.

Ehrenberg A. (dir.), 1999, Drogues et médicaments psychotropes. Le trouble des frontières, Paris, Esprit.

Fainzang S., 2001, Médicaments et société. Le patient, le médecin et l'ordonnance, Paris, Presses Universitaires de France.

Foucault M., 1994, "Les techniques de soi", In Defert, D. et Ewald, F. (dir.), Dits et Ecrits, Paris, Gallimard, pp.789-813.

Haxaire C., Brabant Hamonic J. et Cambon E., 1999, "C'était pas comme un drogue si vous voulez, mais enfin"., In Ehrenberg, A., op. cit., pp.171-208.

Homedes N. et Ugalde A., 2001, Improving the use of pharmaceuticals through patient and community level interventions, Social Science $\mathcal{E}$ Medicine, 52, 1, pp.99134.

Lambert B. L., Chang K. Y. et Lin S. J., 2001, Effect of orthographic and phonological similarity on false recognition of drug names, Social Science $\mathcal{E}$ Medicine, 52, 12, pp.1843-1857.

Makoul G., Arntson P. et Schofield T., 1995, Health Promotion in Primary-Care Physician-Patient Communication and Decision-Making About Prescription Medications, Social Science \& Medicine, 41, 9, pp.1241-1254.

Maurain C., 1996, La pluralité des émetteurs de l'information sur le médicament, Cahiers de sociologie et de démographie médicales, 36, 3, pp.209-224.

Ménoret M., 1999, Les Temps du cancer, Paris, Éditions du CNRS.

Meyer P., 1984, La Révolution des médicaments, mythes et réalités, Paris, Fayard. 
Mol A. et Law J., à paraître, Situated Bodies and Distributed Selves : Enacting Hypoglycaemia, Body and Society,

Pignarre P., 1999, Puissance des psychotropes, pouvoir des patients, Paris, PUF.

Pollock K. et Grime J., 2000, Strategies for reducing the prescribing of proton pump inhibitors (PPIs), Social Science \& Medicine, 51, 12, pp.1827-1839.

Rabeharisoa V. et Callon M., 1999, Le pouvoir des malades. L'Association française contre les myopathies et la Recherche, Paris, Presses de l'École des mines.

Willems D., 1995, Mesurer sa respiration - Reconstituer le corps avec un objet médical, Techniques \& Culture, 25-26, pp.55-72. 